\title{
Automatic Segmentation of Non-rigid Objects in Image Sequences Using Spatiotemporal Information
}

\author{
Cheolkon Jung and Joongkyu Kim \\ School of Information and Communication Engineering, Sungkyunkwan University, \\ Suwon 440-746, Republic of Korea \\ ckjung@ece.skku.ac.kr, jkkim@skku.edu
}

\begin{abstract}
This paper provides an automatic segmentation method of non-rigid objects in image sequences. The non-rigid objects have fuzzy, blurred, and indefinite boundaries such as smoke and clouds, and are random and unpredictable in spatial and temporal domains. To segment the non-rigid objects, a new segmentation approach considering random and unpredictable characteristics of the non-rigid objects is needed. In this paper, we propose a new segmentation method of the non-rigid objects in image sequences using spatiotemporal information. The procedure toward complete segmentation consists of three steps: spatial segmentation, temporal segmentation, and fusion of the spatial and temporal segmentation results. By means of experiments on various test sequences, we demonstrate that the performance of our method is quite impressive from the viewpoints of the segmentation accuracy.
\end{abstract}

\section{Introduction}

Recent advances in the internet, high-speed computing, and storage systems have resulted in tremendous interest in digitizing large archives of video data and providing users with interactive access. Due to the shear volume of video data, all these capabilities require an efficient video analysis algorithm that can automatically segment the video objects and index video data. The development of a powerful moving object segmentation algorithm is an important requirement for many computer vision and ubiquitous systems. In video surveillance applications, motion detection can be used to determine the presence of people, vehicles, or other unexpected objects. This initiates more complex activity recognition steps. Segmentation of moving objects in the observed scenes is an important issue in order to solve traffic flow measurements or for behavior detection during sports activities [1-5].

Up to the present, many significant achievements have been made by researchers in the field of the moving object segmentation. Arch and Kaup presented a moving object segmentation method using a statistical approach for video analysis [6]. They modeled the characteristics of pixel difference for background between two consecutive frames, as a Gaussian distribution. For a given level of significance, the resulting threshold value was theoretically obtained and a threshold level was set for the frame difference image, so as to yield a change detection mask (CDM). The CDM was a binary image in which pixel differences exceeding the threshold value were declared 
as being of changed intensity and, otherwise, as being intensity-invariant. Meier and Ngan presented an automatic segmentation method for moving objects using a binary image model to track a moving object [7]. The binary model was derived from an edge image and updated every frame to keep the moving object undergoing changes in its shape and spatial location at a certain instance of time. Detection of a moving object was made based on binary model matching between two consecutive frames using Hausdorff distance. The localization of a moving object relies on binary image matching; temporal coherence of the object is preserved even in the case of discontinuous object motion. Kim et al. presented an automatic segmentation method for moving objects based on spatiotemporal information [8]. The method utilized temporal information for localizing the moving objects and spatial information for the acquisition of precise object boundaries and semantic region partition. A combination method for spatiotemporal segmentation improves segmentation accuracy and temporal coherence of moving object boundaries. This method has been adopted as an automatic segmentation tool as informative in MPEG-4. In addition, there have been edge-based methods, feature-based methods, semiautomatic segmentation methods, and so on $[4,9-11,23]$.

However, these methods do not consider random and unpredictable characteristics of non-rigid objects with large deformation rates over time. A non-rigid object has fuzzy, blurred, and indefinite boundaries such as smoke and clouds, and is random and unpredictable in both spatial and temporal domains as shown in Fig. 1. Therefore, a new approach for the non-rigid object segmentation should be needed.

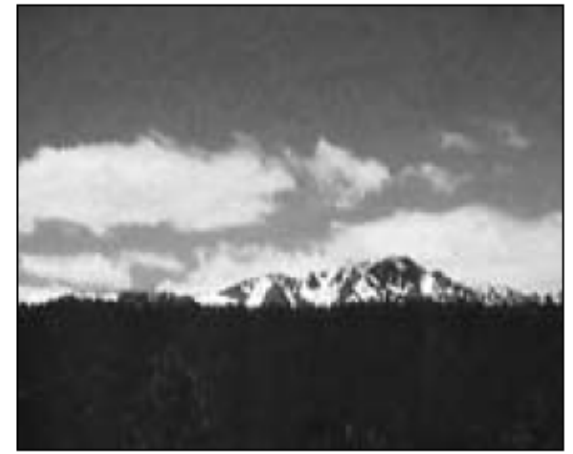

(a)



(b)

Fig. 1. Representative non-rigid objects with large deformation rates over time. (a) Cloud. (b) Smoke.

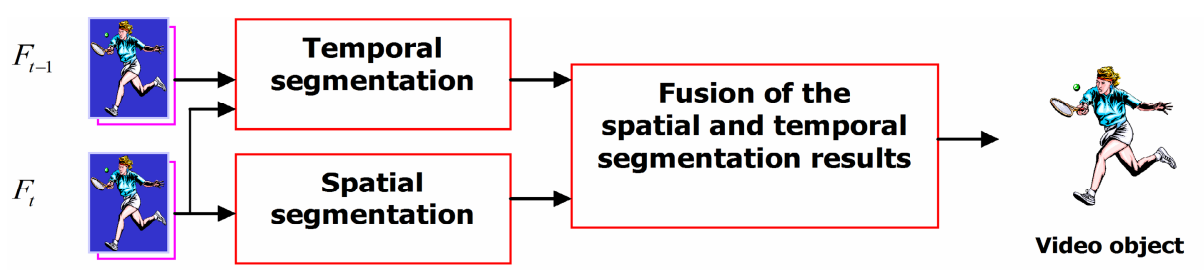

Fig. 2. Flow chart of the proposed algorithm 
In this paper, we present an automatic segmentation algorithm of the non-rigid objects with large deformation rates. Due to the randomness and unpredictability of the non-rigid objects, we make use of a Markov random field (MRF) model in both spatial and temporal domains. The segmentation flow of the presented scheme is shown in Fig. 2, where $F_{t-1}$ and $F_{t}$ are previous and current frames, respectively. The scheme consists of three procedures: 'spatial segmentation', 'temporal segmentation', and 'fusion of the spatial and temporal segmentation results'. The spatial segmentation procedure divides the image into semantic regions with precise object boundaries using a MRF model. The temporal segmentation procedure localizes moving regions of objects in the image. Then, the fusion of the spatial and temporal segmentation results produces accurate segmentation results for moving objects. Experimental results show that the presented algorithm achieves for the accurate non-rigid object segmentation.

This paper is organized as follows. Spatial and temporal segmentation are addressed in Sections 2 and 3, respectively. In Section 4, the fusion of the spatial and temporal segmentation results is explained. Section 5 presents experimental results, and we conclude this paper in Section 6.

\section{Spatial Segmentation}

We have used a MRF model for the spatial segmentation. In order to model the intensity of an image as MRF, the correct number of clusters should be determined. If $z(x, y)$ is the intensity of a pixel $(x, y)$, the distribution of $z$ is represented as the sum of $K$ probability density functions (PDFs) in Fig. 3. Assuming these PDFs are Gaussian, the mixture density model takes the equation $(1)[12,24]$.

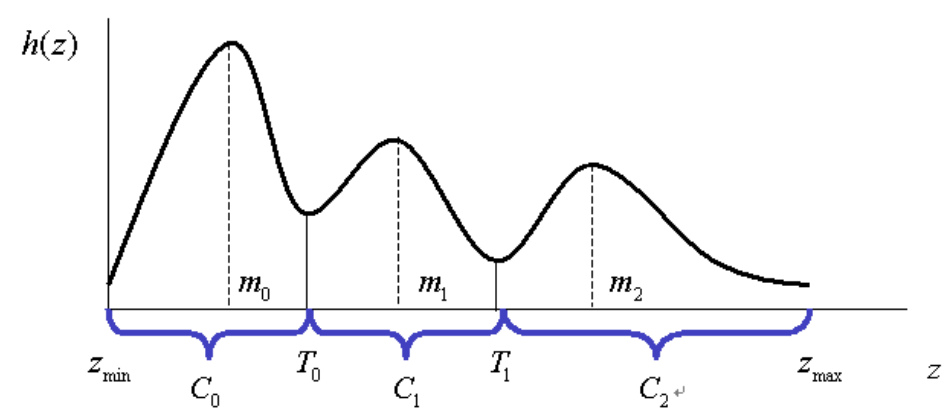

Fig. 3. Distribution of $z$

$$
h(z)=\sum_{l=0}^{K-1} \frac{P_{l}}{\sigma_{l} \sqrt{2 \pi}} \exp \left[-\frac{1}{2}\left(\frac{z-m_{l}}{\sigma_{l}}\right)^{2}\right]
$$

where $P_{l}$ denotes the a priori probability of the particular mode such that $\Sigma P_{l}=1$, and $m_{l}$ and $\sigma_{l}$ denote the mean and the standard deviation of each mode, respectively. Since the number of PDFs is equal to the number of clusters, we have used the cluster 
validity measure presented by Rose to determine the number of clusters, $K$. The idea of this method is that optimal $K$ minimizes within-cluster scatter and maximizes the between-cluster separation [13]. The improved cluster validity measure validity is defined as

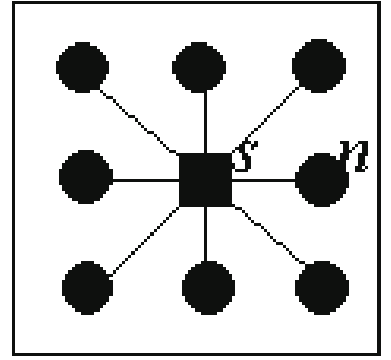

(a)

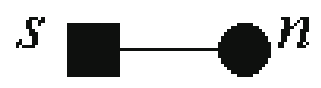

(b)

Fig. 4. (a) Neighborhood system. (b) A binary click.

$$
\text { validity }=w \cdot \frac{\frac{1}{N} \sum_{l=0}^{K-1} \sum_{z \in C_{l}}\left|z-m_{l}\right|}{\frac{1}{K-1} \sum_{l=1}^{K-1}\left|m_{l}-m_{l-1}\right|} .
$$

where $w$ is weighted constants, $N$ is the number of pixels in the image, and $C_{l}$ is the $l$ 'th cluster $(l=0,1, \ldots, K-1)$. The optimal $K$ is the value which minimizes the validity. To find the cluster of a pixel, we should determine the optimal threshold value $T_{n}$. If we assume that $\sigma=\sigma_{0}=\cdots=\sigma_{K-1}$, the optimal threshold value $T_{n}$ is given in [14]

$$
T_{n}=\frac{m_{n}+m_{n+1}}{2}+\frac{\sigma^{2}}{m_{n}-m_{n+1}} \ln \frac{P_{n+1}}{P_{n}} .
$$

If $P_{n}$ is equal to $P_{n+1}$, the optimal threshold is simply the average of the two class means. By equation (3), we can assign the cluster label to each pixel. If the label field of each pixel is $L(x, y)$, the label $l$ is equal to the index of cluster $C_{l}$ as follows.

$$
L(x, y)=l, \quad z(x, y) \in C_{l}
$$

Since the initial label $l$ is determined by equation (4), we can merge regions with spatial homogeneity using the MRF model. Neighborhood system $N$ and binary cliques at each pixel $(x, y)$ are defined as shown in Fig. 4. If we regard a random field $z$ as MRF, the probability of $z$ is given by a Gibbs distribution that has the following form according to the Hammersley-Clifford theorem [10, 15].

$$
P(z)=Q^{-1} \times e^{-U(z)}
$$


where $Q$ is a constant called the partition function, and $U(z)$ is the energy function. We can find the label $l^{*}$ that the posteriori probability $P(l \mid z)$ is maximal. By using the maximum a posteriori criterion (MAP), the label $l^{*}$ is obtained by equation (6).

$$
l^{*}=\arg \max _{l} P(l \mid z) .
$$

By Bayes' rule, the relationship between $z$ and $l$ is expressed as

$$
P(l \mid z) \propto P(z \mid l) P(l) .
$$

where $P(l \mid z)$ is a conditional probability of $l$ in dependence on $z$, and $P(l)$ is a priori probability of $l$. Therefore, we can express equation (6) as the following form.

$$
l^{*}=\arg \max _{l}(P(z \mid l) \cdot P(l))
$$

From equation (5), we get:

$$
\max _{l}(P(l \mid z))=\min _{l}(U(l \mid z))
$$

Then, the maximization of the a posteriori probability is equivalent to the minimization of the energy function $U$. The energy function is classically the sum of two terms (corresponding to data-link and prior knowledge, respectively) [10]:

$$
U(l \mid z)=U_{a}(z \mid l)+U_{m}(l)
$$

The link-to-data energy $U_{a}(z \mid l)$ (attachment energy) is expressed as

$$
U_{a}(z \mid l)=\frac{1}{2 \sigma^{2}} \sum_{(x, y)}\left[z-m_{l}\right]^{2} .
$$

where $\sigma^{2}$ is the observation variance.

The model energy $U_{m}(l)$ is a regularization term, and puts a priori constraints on the masks of moving objects, erasing isolated points due to noise. Its expression is given by

$$
U_{m}(l)=\sum_{c} V_{c}\left(l_{s}, l_{n}\right)
$$

where $c, s$, and $n$ denote a binary clique, a current pixel, and pixel of a neighbor, respectively. $l_{s}$ is a label of $s, l_{n}$ is a label of $n$, and $V_{c}\left(l_{s}, l_{n}\right)$ is a potential function associated with a binary clique, $c=(s, n)$. To put homogeneity constraints into the model, it is defined as:

$$
V_{c}\left(l_{s}, l_{n}\right)= \begin{cases}-\beta, & \text { if } l_{s}=l_{n} \\ +\beta, & \text { if } l_{s} \neq l_{n}\end{cases}
$$

where the positive parameter $\beta$ depends on the nature of the clique. 
In order to find the minimum of the energy function, ICM (iterated conditional modes) is used [16]. For each pixel $s$ of the current image, the labels from 0 to $K-1$ are tested, and the label that induces the minimum local energy in the neighborhood is kept. The process iterates over the image until convergence. Suppose the label of a current pixel in iteration $k$ is denoted as $l^{k}$ and a prescribed small number is $\varepsilon$. The fixed label of each pixel is achieved if the following condition is satisfied [17]:

$$
\sum_{(x, y)}\left|l^{k}-l^{k-1}\right|<\varepsilon
$$

\section{Temporal Segmentation}

In the temporal segmentation procedure, moving parts of objects are localized in sequential images. Using the temporal segmentation procedure, we can find mobile regions in spatially segmented regions. Optical flow is the distribution of apparent velocities of movement of brightness patterns in an image. Let $I$ be the intensity of a pixel $(x, y)$ of an image in time $t$. The optical flow constraint equation can be expressed as $[12,18,22]$ :

$$
I_{x} u+I_{y} v+I_{t}=0
$$

where $u$ and $v$ are two components of the velocity vector, and $I_{x}, I_{y}, I_{t}$ are partial derivatives about $x, y, t$, respectively. To compute $u$ and $v$, we use the method presented by Lucas and Kanade [12]. This approach assumes that the motion vector remains unchanged over a particular block of pixels denoted by $B$. Under this assumption, the velocity vector is computed as follows:

$$
\left[\begin{array}{l}
u \\
v
\end{array}\right]=\left[\begin{array}{ll}
\sum_{X \in B} I_{x} I_{x} & \sum_{X \in B} I_{x} I_{y} \\
\sum_{X \in B} I_{x} I_{y} & \sum_{X \in B} I_{y} I_{y}
\end{array}\right]^{-1}\left[\begin{array}{l}
-\sum_{X \in B} I_{x} I_{t} \\
-\sum_{X \in B} I_{y} I_{t}
\end{array}\right] .
$$

The output of each pixel in images is binary: the motion exists or does not. The situation where motion exists is denoted as $s_{1}$, and the opposite, stationary, situation is denoted as $s_{0}$ [19]. Let random variable $r$ be the magnitude of the velocity vector. Then, $r$ is defined as:

$$
r=\sqrt{u^{2}+v^{2}}
$$

We have modeled $r$ as a Gaussian random variable. If $a_{0}$ is the mean of $r$ when motion does not exist and $a_{l}$ is the mean when motion exists, the random variable, $r$, has the two conditional probability density functions (PDFs), $p\left(r \mid s_{0}\right), p\left(r \mid s_{1}\right)$, with mean value of $a_{0}$ and $a_{1}$, respectively, as shown in Fig. 5. 


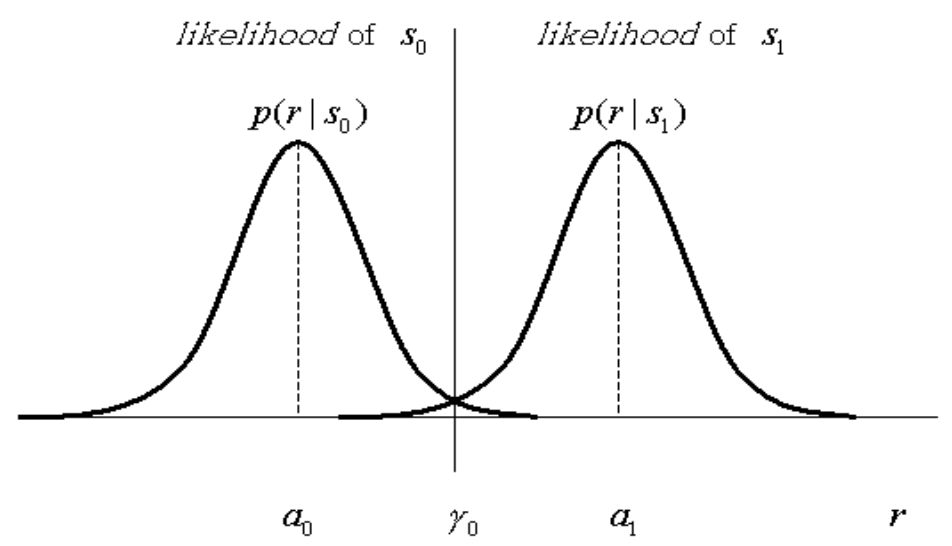

Decision line

Fig. 5. Conditional probability density function: $p\left(r \mid s_{0}\right), p\left(r \mid s_{1}\right)$

These PDFs are represented as [20]:

$$
\begin{aligned}
& p\left(r \mid s_{0}\right)=\frac{1}{\sigma \sqrt{2 \pi}} \exp \left[-\frac{1}{2}\left(\frac{r-a_{0}}{\sigma}\right)^{2}\right] \\
& p\left(r \mid s_{1}\right)=\frac{1}{\sigma \sqrt{2 \pi}} \exp \left[-\frac{1}{2}\left(\frac{r-a_{1}}{\sigma}\right)^{2}\right]
\end{aligned}
$$

where, $\sigma^{2}$ is a variance of noise.

In order to optimize the binary decision threshold for deciding motion existence, we use the minimum error criterion for equally likely binary signals corrupted by Gaussian noise. The threshold level, $r_{0}$, is represented by $\left(a_{0}+a_{1}\right) / 2$. Using the threshold level, we can assign a label $l$ to each pixel. We assign the label 1 to a pixel where motion exists and the label 0 to a pixel where it does not. The label is obtained as follows:

$$
l=\left\{\begin{array}{ll}
1, & \mathrm{r} \geq \gamma_{0} \\
0, & \mathrm{r}<\gamma_{0}
\end{array} .\right.
$$

When we decide the presence of motion by $r_{0}$, two types of errors occur. The first type is that we make a decision on motion, when motion does not exist, and this error is called the false alarm. The second type is that we make a decision on non-motion, when motion actually exists, and this error is called the miss. We make use of the MRF model based on Bayes' rule to resolve these two types of errors $[10,15]$. 


\section{Fusion of the Spatial and Temporal Segmentation Results}

By using the fusion module that combines the spatial segmentation result and temporal segmentation result, moving regions are discriminated from background regions. Through connected component labeling, we assign the proper label to each spatially segmented region [21]. Then, $R_{i, t}^{\text {proj }}$ is projected region on top of the spatially segmented region $R_{i, t}$ by the temporal mask $T M_{t}$ obtained from temporal segmentation between the previous frame $F_{t-1}$ and the current frame $F_{t}$. Let $N\left(R_{i, t}{ }^{\text {proj }} \cap R_{i, t}\right)$ be the number of pixels within the intersection $R_{i, t}{ }^{\text {proj }} \cap R_{i, t}$ of the two regions $R_{i, t}{ }^{\text {proj }}$ and $R_{i, t}$. A decision rule whether or not $R_{i, t}$ is a moving region is defined as:

$$
P=\frac{N\left(R_{i, t}^{\text {proj }} \cap R_{i, t}\right)}{N\left(R_{i, t}\right)}\left\{\begin{array}{lr}
\geq \tau: & \text { moving region } \\
<\tau: & \text { background region }
\end{array} .\right.
$$

where $N\left(R_{i, t}\right)$ is the number of pixels in $R_{i, t}$. If the value of $P$ is greater than or equal to a given threshold $\tau$, the whole region $R_{i, t}$ is considered as a moving region; otherwise a background region [8]. Here, the value $\tau$ was determined by experiments.

\section{Experimental Results}

The proposed segmentation algorithm has been experimentally investigated by means of computer simulations. First, four test sequences, Table tennis, Foreman, Street, and Smoke with the QCIF format $(176 \times 144)$, were used in the experiment (Fig. 6). Table tennis and Foreman sequences have non-rigid objects with small deformation rates, and Street and Smoke sequences with large deformation rates.

In the spatial segmentation procedure, the intensity field in an image is regarded as MRF, and segmented by energy minimization. We should determine the number of clusters before energy minimization. Table 1 shows the validity for each $K$. The optimal number $K$ is 9 for the Table tennis sequence, 10 for the Foreman sequence, 6 for the Street sequence, and 7 for the Smoke sequence. By using the optimal $K$, we can find averages and thresholds for labeling at each point in the image. Then, we can segment an image by energy minimization. Fig. 7 shows the results of the spatial segmentation.

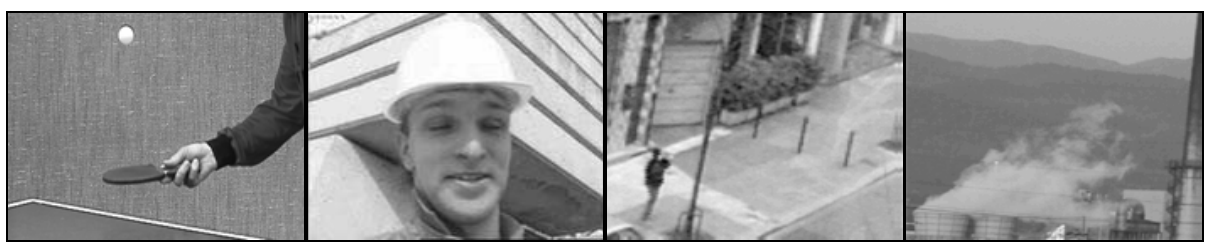

(a)

(b)

(c)

(d)

Fig. 6. Original images. (a) Table tennis. (b) Foreman. (c) Street. (d) Smoke. 
Table 1. Validity

\begin{tabular}{c|c|c|c|c}
\hline \hline$K$ & Table tennis & Foreman & Street & Smoke \\
\hline \hline 2 & 0.04232 & 0.06757 & 0.07306 & 0.02579 \\
\hline 3 & 0.01685 & 0.03920 & 0.04285 & 0.01956 \\
\hline 4 & 0.01880 & 0.02463 & 0.04593 & 0.01784 \\
\hline 5 & 0.01393 & 0.01694 & 0.02706 & 0.02286 \\
\hline 6 & 0.00854 & 0.02188 & $\mathbf{0 . 0 1 9 0 8}$ & 0.02060 \\
\hline 7 & 0.00777 & 0.02499 & 0.02095 & $\mathbf{0 . 0 0 5 8 3}$ \\
\hline 8 & 0.00650 & 0.02541 & 0.02345 & 0.00591 \\
\hline 9 & $\mathbf{0 . 0 0 5 3 5}$ & 0.01831 & 0.02160 & 0.00773 \\
\hline 10 & 0.21581 & $\mathbf{0 . 0 1 6 1 8}$ & 0.02150 & 0.00890 \\
\hline \hline
\end{tabular}

While the spatial segmentation procedure proceeds, the temporal segmentation procedure is performed on consecutive frames. In the temporal segmentation procedure, the velocity vector is computed at each pixel by optical flow analysis, and then the existence of motion is determined by the velocity vector. The size of a particular block $B$ is $3 \times 3, \varepsilon$ is 1 , and $\sigma$ is 100 . The iteration number $k$ varies from $5 \sim 15$. Fig. 8 shows the results of the temporal segmentation. As shown in this figure, black and white pixels are non-motion and motion pixels, respectively. Here, the label where motion exists is assigned 1 , and the label where motion does not exist is assigned 0 .

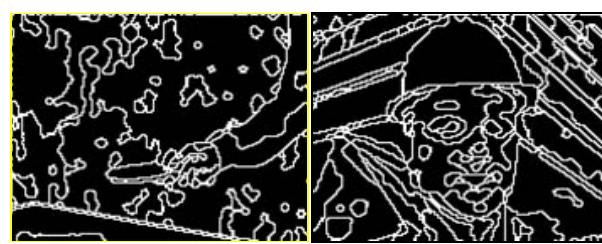

(a) (b)

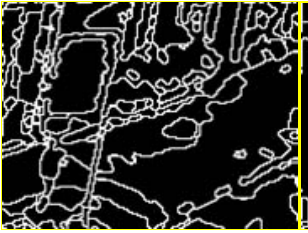

(c)

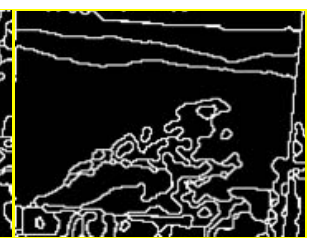

(d)

Fig. 7. Spatial segmentation results. (a) Table tennis. (b) Foreman. (c) Street. (d) Smoke.

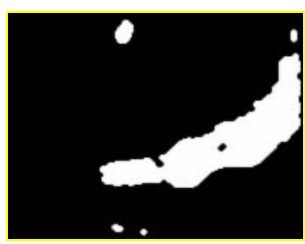

(a)

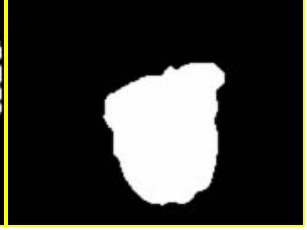

(b)

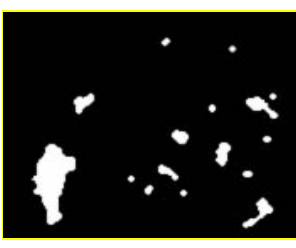

(c)

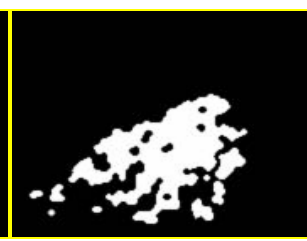

(d)

Fig. 8. Temporal segmentation results. (a) Table tennis. (b) Foreman. (c) Street. (d) Smoke. 
After the spatial and temporal segmentation procedures are performed, both segmentation results are combined to yield final segmentation results. Fig. 9 shows the final segmentation results. Moving objects are captured by the temporal segmentation procedure and the spatial segmentation results precisely represent the object boundaries. These results show that the presented algorithm has good performance in automatic segmentation of the non-rigid objects.

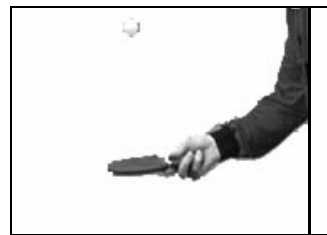

(a)

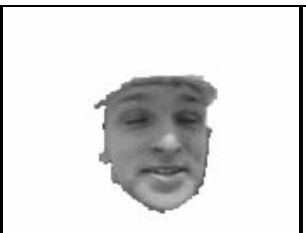

(b)

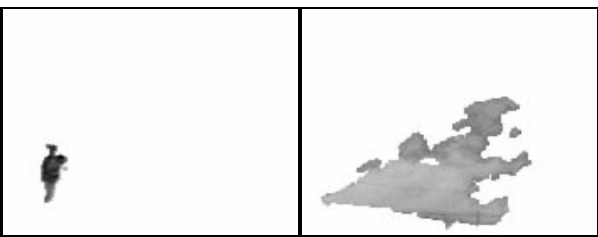

(c) (d)

Fig. 9. Final segmentation results. (a) Table tennis. (b) Foreman. (c) Street. (d) Smoke.
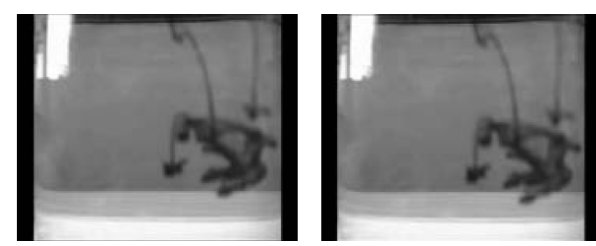

(a)



(b)

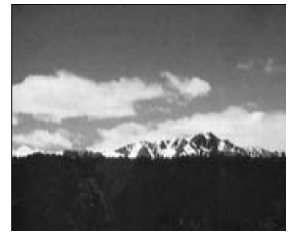

Fig. 10. Some other non-rigid objects. (a) Diffusion. (b) Cloud.

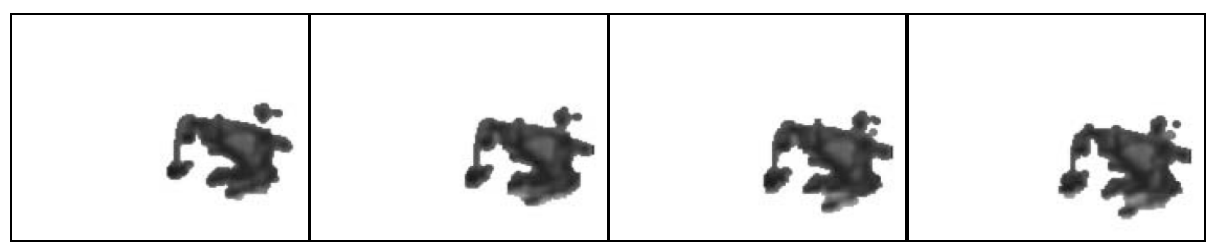

(a)

(b)

Fig. 11. Final segmentation results of the Diffusion sequence. (a) $450^{\text {th }}$ frame. (b) $465^{\text {th }}$ frame. (c) $480^{\text {th }}$ frame. (d) $495^{\text {th }}$ frame.

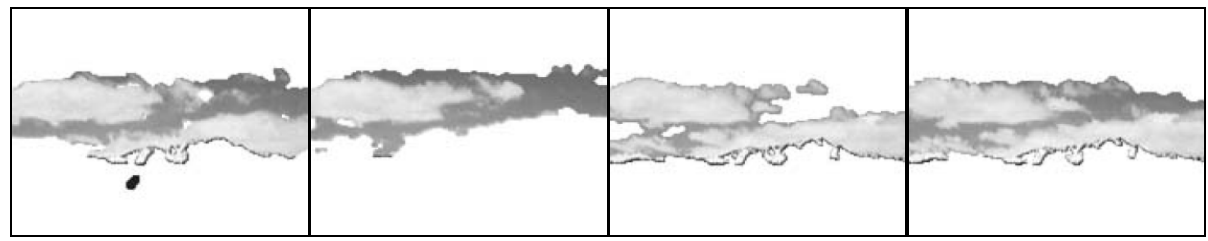

Fig. 12. Final segmentation results of the Cloud sequence. (a) $7^{\text {th }}$ frame. (b) $8^{\text {th }}$ frame. (c) $9^{\text {th }}$ frame. (d) $10^{\text {th }}$ frame. 
Experiments are performed on some other non-rigid objects: cloud (Cloud) and diffusing dye (Diffusion) as shown in Fig. 10. In the Diffusion sequence, the water-soluble dye diffuses in water. We have captured 30 frames per second using a digital camcorder (Sony DCR-TRV20). Since the diffusing dye is the object with a fuzzy, blurred, and indefinite boundary, it would be considered as a non-rigid object. In the Cloud sequence, the cloud floats around a mountain; this object is also a non-rigid object because the shape changes much over time. 6 frames per hour are captured in this sequence since cloud movement is slow. Figs. 11 and 12 show the segmentation results for the Diffusion and Cloud sequences, respectively. The diffusing dye in Fig. 11 is well segmented even though the object shape deforms over time. Also, segmented results for the cloud (Fig. 12) satisfactorily track its deforming shape over time.

\section{Conclusions}

In this paper, we present an automatic segmentation algorithm of moving non-rigid objects in image sequences. The segmentation of foreground moving objects from the background is very useful in many contexts. These include domains such as video surveillance, traffic flow measurements, behavior detection, and object based video coding. We designed a robust algorithm for foreground segmentation that combines temporal segmentation using optical flow analysis. We considered random and unpredictable characteristics of the non-rigid objects using a MRF model in both spatial and temporal domains. We carried out various experiments of non-rigid objects, such as smoke, clouds, and diffusing dye. Experimental results show that the presented algorithm performs well in segmenting the non-rigid objects with large deformation rates over time.

Practical applications of the proposed segmentation algorithm would be observation part of the weather forecast using satellite scenes, medical part of the diagnosis of a skin disease, defense part of the watching system using infrared scenes, chemical part of the flow measurement of a gas or bubbles, and environmental part of the analysis of environmental pollution.

\section{Acknowledgement}

An initial version of this paper appeared in the International Conference on Signal Processing (ICSP) [25]. The authors would like to thank the anonymous ICSP and PSIVT reviewers for their valuable comments and suggestions.

\section{References}

1. Spagnolo, P., Orazio, T.D., Leo, M., Distante, A.: Moving object segmentation by background substraction and temporal analysis. Image and Vision Computing 24, 411-423 (2006)

2. Kuo, M., Hsieh, C.H., Huang, Y.R.: Automatic extraction of moving objects for headshoulder video sequences. Journal of Visual Communication and Image Representation 16, 68-92 (2005)

3. Dimitrova, N., Zhang, H.J., Shahraray, B., Sezan, I., Zakhor, A., Huang, T.: Applications of video content analysis and retrieval. IEEE Multimedia 9, 43-55 (2002) 
4. Fan, J., Yu, J., Fujita, G., Onoye, T., Wu, L., Shirakawa, I.: Spatiotemporal segmentation for compact video representation. Signal Processing: Image Communication 16, 553-566 (2001)

5. Kim, M.C., Jeon, J.G., Kwak, J.S., Lee, M.H., Ahn, C.: Moving object segmentation in video sequences by user interaction and automatic object tracking. Image and Vision Computing 19, 245-260 (2001)

6. Aach, T., Kaup, A.: Bayesian algorithms for adaptive change detection in image sequences using Markov random fields. Signal Processing: Image Communication 7, 147-160 (1995)

7. Meier, K., Ngan, N.: Automatic segmentation of moving objects for video object plane generation. IEEE trans. Circuits and Systems for Video Technology 8(5), 525-538 (1998)

8. Kim, M.C., Choi, J.G., Kim, D., Lee, H., Lee, M.H., Ahn, C., Ho, Y.S.: A VOP generation tool: automatic segmentation of moving objects in image sequences based on spatiotemporal information. IEEE Trans. Circuits and Systems for Video Technology 9 (1999)

9. Jung, C., Kim, K.S., Kim, J.K.: Automatic moving object segmentation using automatic region growing algorithm. Journal of Korea Information and Communications Society 26, 187-193 (2001)

10. Luthon, F., Caplier, A., Lievin, M.: Spatiotemporal MRF approach to video segmentation: Application to motion detection and lip segmentation. Signal Processing 76, 61-80 (1999)

11. Grinias, I., Tziritas, G.: A semi-automatic seeded region growing algorithm for video object localization and tracking. Signal Processing: Image Communication 16, 977-986 (2001)

12. Tekalp, A.M.: Digital video processing. Prentice Hall, Englewood Cliffs (1995)

13. Ray, S., Turi, R.H.: Determination of number clusters in K-means clustering and application in colour image segmentation. In: Proc. of ICAPRDT 1999, pp. 137-143 (1999)

14. Gonzalez, R.C., Woods, R.E.: Digital image processing, pp. 443-458. Addison Wesley, Reading (1992)

15. Zimanyi, M.: Reconstruction of tomographic data by Markov random fields. In: Proc. of Central European Seminar on Computer Graphics (1998)

16. Dubes, R.C., Jain, A.K., Nadabar, S.G., Chen, C.C.: MRF model-based algorithms for image segmentation. In: Proc. of ICPR (10 th International Conference on Pattern Recognition), vol. 1, pp. 808-814 (1990)

17. Wei, J., Li, Z.: An efficient two-pass MAP-MRF algorithm for motion estimation based on mean field theory. IEEE Trans. on Circuits and Systems for Video Technology 9, 960-972 (1999)

18. Horn, B.K.P., Schunck, B.G.: Determining optical flow. Artificial Intelligence 17, 185$203(1981)$

19. Barkat, M.: Signal detection \& estimation, pp. 115-174. Artech House (1991)

20. Sklar, B.: Digital commnication, pp. 132-138. Prentice Hall, Englewood Cliffs (1988)

21. Jain, R., Kasturi, R., Schunck, B.G.: Machine vision, pp. 25-72. McGraw-Hill, New York (1995)

22. Jung, C., Kim, J.K.: Motion segmentation using Markov random field model for accurate moving object segmentation. In: Proc. of ACM ICUIMC 2008, pp. 414-418 (2008)

23. Zitnick, L., Jojic, N., Kang, S.B.: Consistent segmentation for optical flow estimation. In: Proc. ICCV 2005, pp. 1308-1315 (2005)

24. Chen, J., Tang, C.K.: Spatio-temporal markov random field for video denosing. In: Proc. of IEEE CVPR 2007, pp. 1-8 (2007)

25. Jung, C., Kim, J.K.: Non-rigid object segmentation in video sequences using Markov random field. In: Proc. of ICSP 2002 (6th International Conference on Signal Processing), vol. 1, pp. 624-627 (2002) 